\title{
Metasurface sensors for healthcare applications
}

\author{
Antoine Durant ${ }^{1,2}$, Célia Lacoste ${ }^{1,3}$, Erin Donnelly ${ }^{1}$, Luigi La Spada ${ }^{1}$ \\ ${ }^{1}$ School of Engineering and the Built Environment, Edinburgh Napier University, Edinburgh, United Kingdom \\ ${ }^{2}$ IUT1, Université Grenoble Alpes, Grenoble, France \\ ${ }^{3}$ INP-ENSEEIHT, University of Toulouse, Toulouse, France \\ 1.laspada@napier.ac.uk
}

\begin{abstract}
Electromagnetic sensors have received huge attention in the last few years, due to the rising demand of devices able to improve quality, performance and safety in different industrial sectors: both sensing and medical industries are outstanding examples. Despite reliable diagnostic technologies have been developed, some drawbacks are still present: bandwidth, large dimensions and limited response control. Metasurfaces, bi-dimensional engineered materials, represent an optimal solution to overcome such issues enhancing existing systems for an accurate diagnosis. Therefore, in this paper, metasurface-based sensors are proposed and realized by using new additive manufacturing processes. The structures are finally experimentally tested and verified in different medical diagnostic applications, namely: cancer stage recognition, glucose/sugar levels measurements and blood oxygen saturation detection. The high performances shown by such meta-sensors, in terms of selectivity and sensibility, pave a new way to realize advanced platforms for non-invasive, high quality and faster patient diagnosis.
\end{abstract}

Index Terms-metasurfaces, sensors, medical diagnostics, additive manufacturing, healthcare.

\section{INTRODUCTION}

The study of electromagnetic sensors and the possibility to control and manipulate the properties of such waves at will (i.e. amplitude, phase, frequency response and polarization) has been a hot topic in the last decades. Of great interest, in the last few years, it is their use in practical applications such as telecommunications, automotive, computer science, and medical diagnostics [1]. The aging and expanding population, increasing the demand for an efficient and fast medical follow-up, are accelerating the development of reliable and accurate real-time diagnostic tools [2]. Several technologies have been used to this regard showing both interesting advantages (i.e. simplicity and speed of analysis, integration, immunity to external disturbances, high sensitivity and selectivity, and the possibility of mass production) and limitations (relatively large dimensions, high losses and scalability) [3]. New engineered surface materials called metasurfaces have been implemented to overcome such issues [4]. They derive their extraordinary properties (i.e. sharp resonant peaks, narrowbandwidth, losses) from both material and geometrical characteristics. Even though such properties are clearly detrimental for many applications, they are extremely useful for sensing and medical diagnostics [5]: (i) sharp resonant peaks ensures high sensitivity; (ii) narrow-bandwidth permits to have high selectivity properties; (iii) losses can be used to enhance and boost sensors performances to obtain strong localization, confinement and enhancement of the electromagnetic field for a more reliable sensing. The main advantage in using them as sensors is the possibility to design and tune such properties at will, and in doing so, in the case of medical diagnosis, to target and detect specific molecules or compounds. This is what will be address in this paper by developing a rigorous approach to model, design and manufacture metasurfaces for medical diagnostics. Such a technique creates a robust link between arbitrary shape surfaces and their analogue material properties in an intuitive manner. The paper is structured as follows: (i) the modeling theory links the incident electromagnetic wave properties to the field phenomena in the structure, useful to understand its EM response. The design section links the field phenomena to the geometrical parameters of the structure, a crucial step to obtain the full control on the sensor response and tune it accordingly to the required application/target; (ii) then, new and inexpensive Additive Manufacturing techniques will be used to realize multi-purpose sensors; (iii) finally, the realized sensing platforms will be used for medical diagnostics applications such cancer stage recognition, glucose/sugar monitoring and blood oxygen saturation detection.

Results will show how metasurfaces are highly promising structures for non-invasive healthcare monitoring applications, transforming and improving our everyday life quality.

\section{MATHERIALS \& METHODS}

Fig.1(a) shows the geometry of a metasurface formed by metallic (dielectric) inclusions (whose unit-cell is depicted in Fig.1(b)), printed on a grounded dielectric slab with thickness $t$, relative permittivity $\varepsilon_{\text {slab }}$ and magnetic permeability of free space $\mu_{\text {slab }}=\mu_{0}$. In [6] it has been demonstrated that (electrically) ultrathin metallic/dielectriconly metasurfaces are not ideal structures for practical applications. For this reason, in this work, metal-dielectric metasurfaces with finite (still small) thickness [7] are considered. With this assumption, we can study the structure of Fig.1(a) with a multi-layer model as shown in Fig. 1(c): the metasurface with non-homogeneous permittivity $\varepsilon(\mathbf{r})$ and/or permeability $\mu(\mathbf{r})$ depending on the vector position $\mathbf{r}$; the substrate as a homogeneous dielectric with permittivity $\varepsilon_{\mathrm{r}}$; a metallic ground plane acting as a perfect electric conductor (PEC). 


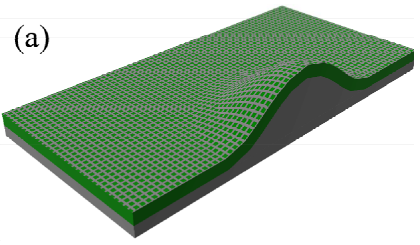

(b)

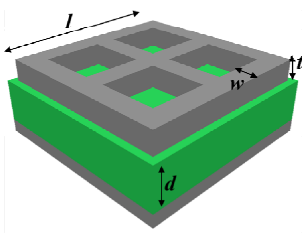

(c)

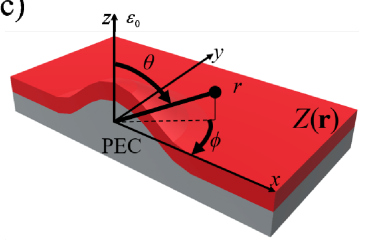

(d)

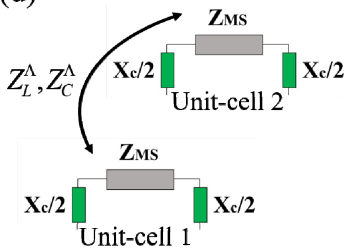

Fig. 1. (a) Generic curvilinear metasurface with grounded dielectric slab underneath; (b) Planar view of metasurface unit-cell; (c) Multi-layer model for metasurface depicted in (a); (d) Metasurface equivalent circuit model.

\section{A. Modeling \& Design of MetaSurface sensors}

The aim of the modeling step is to link the wave properties (amplitude and phase) with the structure field phenomena (electric $\mathbf{E}$ and magnetic $\mathbf{H}$ ) through its impedance distribution $\mathrm{Z}$ (permittivity and permeability). We need to solve the appropriate wave equation to find both electric and magnetic field components. Such solutions are well known in literature for homogeneous materials [8]. On the contrary for non-homogeneous media, like the metasurface used here, equations (and solutions) became more complex [9]:

$$
\left\{\begin{array}{l}
\nabla^{2} \mathbf{E}+\nabla[\mathbf{E} \cdot \nabla \log \varepsilon(\mathbf{r})]+\omega^{2} \mu_{r} \varepsilon(\mathbf{r}) \mathbf{E}=0 \\
\nabla^{2} \mathbf{H}+\frac{1}{\varepsilon(\mathbf{r})}[\nabla \varepsilon(\mathbf{r})] \times(\nabla \times \mathbf{H})+\omega^{2} \mu_{r} \varepsilon(\mathbf{r}) \mathbf{H}=0
\end{array}\right.
$$

Knowing the electric and magnetic configuration of the structure, its equivalent impedance can be easily evaluated as [10]:

$$
\mathbf{Z}_{\text {field }}(\mathbf{r})=\frac{\mathbf{E}(\mathbf{r})}{\mathbf{H}(\mathbf{r})}
$$

where $\boldsymbol{l}$ is the line chosen element, $(\mathrm{a}, \mathrm{b})$ the electric potential points and $\mathrm{C}$ the closed magnetic loop. The impedance $Z$ in (2) fully describe the metasurface structure properties, allowing the full control of its response.

Since we are interested in real-life curvilinear metasurfaces, we can model the structure by using spherical coordinates $(r, \theta, \phi)$. Therefore, the Impedance in (2) reads:

$$
\mathbf{Z}(\mathbf{r})=\left[1-\left(\frac{2}{\pi} r^{2}\right), \frac{1}{\sin ^{2}(\theta)}, \varepsilon_{r}\right]
$$

The aim of the design step is to obtain the relation between the field phenomena, described by the structure $Z$, and its physical properties: substrate thickness, inclusions dimensions (length 1 , width $\mathrm{w}$, gap g and thickness $\mathrm{t}$ ) and their spatial periodicity $\Lambda$. Each field component has its counterpart in the related lumped elements circuit model.

When a wave is impinging on the metallic (dielectric) patches, both electric $\mathbf{E}$ and magnetic $\mathbf{H}$ field components excite induced electric/magnetic density currents $\mathbf{J}$. The transverse electric (magnetic) currents will flow with high intensity along the metallic (dielectric) layer of the patch; the transverse magnetic (electric) currents will be very weak in the same region [6]. The equivalent unit-cell circuit impedance reads:

$$
Z_{\text {unit-cell }}=\frac{V_{\text {unit-cell }}}{I_{\text {unit-cell }}}=\frac{\int_{a}^{b} \mathbf{E} \bullet d \mathbf{l}}{\oint_{C} \mathbf{H} \bullet d \mathbf{l}}=\frac{1-\omega^{2} L_{t o t} C_{t o t}}{j \omega C_{t o t}}
$$

An ultrathin metallic (dielectric) metasurface can only introduce a discontinuity on the transverse magnetic (electric) component, resulting in a very limited efficient structure [7]. To fully satisfy all the boundary conditions, a further discontinuity on the transverse electric $\mathbf{E}$ (magnetic H) field component is in need: a thicker metasurface or a supporting layer (dielectric substrate). This introduces in the circuit model a reactive element in series, leading to the new Impedance $Z$ :

$$
Z_{\text {tot }}=\frac{Z_{\text {unit-cell }} X_{\text {thickness }}}{Z_{\text {unit-cell }}+2 X_{\text {thickness }}}
$$

In literature adjacent unit-cells are considered sufficiently separated each other, so that any coupling phenomena can be neglected [11]. Here, on the contrary, both intra- and interlayer coupling fields play an important role: highly confine signals, multi and broad -band responses, enhancement of both selectivity and sensibility. Such electric (magnetic) fields across the elements introduce, in the final circuit model, both mutual capacitances (inductances):

$$
Z_{\text {mutual }}^{C}=\varepsilon_{\text {sub }} l \frac{K\left(\sqrt{1-k^{2}}\right)}{K(k)}
$$

Once that all the field phenomena have been described by proper circuit elements, we can finally relate them to the structure physical dimensions (geometry and size), by using formulas coming from electrostatics [12] and magnetostatics $[13,14]$. The final Impedance circuit model is depicted in Fig.1(d). Let's consider the unit-cell depicted in Fig.1(b): a metallic square-shape particle deposited on a homogeneous dielectric substrate. Its field impedance $Z(\mathbf{r})$ is described in (3). By using formulas in $[13,14]$, the total capacitance is the contribution of three different terms, representing a specific sensing area: $\mathrm{C}_{\text {tot }}=\mathrm{C}_{\mathrm{g}}(w, t, g)+\mathrm{C}_{\mathrm{f}}(w, g)+\mathrm{C}_{\mathrm{s}}(l, w, t, g)$, where

(i) in the gap/cavity area $C_{g}$ localized and resonant phenomena create a strong interaction with the target [15]:

$$
C_{g}(w, t)=\frac{\eta}{\sqrt{\varepsilon_{\text {sample }}}} \sqrt{1-\frac{\sin ^{2} \theta_{i}}{\varepsilon_{\text {sample }}}} \tan \left(\beta_{\text {sample }} \sqrt{\varepsilon_{\text {sample }} t}\right)
$$

(ii) The substrate $C_{f}$ is an extremely sensitive area with high Q factor [16]: 
$X_{\text {thickness }}^{-1}=j \omega C_{f}(w, g)=\varepsilon_{\text {sample }} \frac{2 w+\sqrt{2} g}{\pi} \cosh ^{-1}\left[\frac{2 w+g}{g}\right]$

(iii) In the surface area $C_{s}$ the energy is localized at the interface in the form of surface waves, crucial for tiny targets [17]:

$$
C_{s}(l, w, g, t)=2 \varepsilon_{\text {sample }} \frac{w+t}{\pi} \log \left(\frac{8 l}{\pi g}\right)
$$

Similar results can be obtained for Inductive terms.

In the next paragraph we will choose and manufacture such sensing areas accordingly to the target and application required.

\section{B. Manufacturing MetaStructures}

As dictated by the modeling and design approach, Additive Manufacturing (AM) techniques have been used to realize the metasurface [18].

The morphology and permittivity properties of the substrate (Fig.2(a)) was controlled by using the following three stages: particulate filler preparation, composite production and layer fabrication. The challenge in the particulate filler preparation step was to obtain the mixture with the requested permittivity value, whilst controlling its rheology: dispersion of the fillers, particle size distribution, particulate shape, compatibility with the resin used, curing process, and volume fraction. Ceramic materials were used by the mill machine to generate the powder, composed by micro- and nano- meter particles. In the composite production stage, both the resin and the ceramic filler were pre-processed separately in a vacuum system prior to forming the mixture. Subsequently, the filler was added to the resin under vacuum to reduce the void content. Finally, in the layer fabrication stage, to achieve homogenous electromagnetic properties within the layer, it was necessary for the dispersed powder to remain as a stable suspension during the cure cycle. Once cured, the top surface of the structure was machined to match the desired surface profile.

The conductive grid pattern (unit-cell) is deposited onto High Impact Polystyrene (HIPS) planar sheets using screen printing (Fig.2(b)). The silver ink is printed through a polyester screen mesh; the printed pattern is then applied to the screen by using photo-emulsion. The screen mesh is used to transfer the printed pattern to the substrate: a dragging bar is moved across the screen to fill the open mesh apertures with ink. On the reverse stroke, a polyurethane tipped squeegee blade deforms the screen into contact with the substrate momentarily along a line of contact. This causes the ink to wet the sheet and be pulled out of the mesh apertures when the screen recoils after the blade has passed.

Once the conductive grid is deposited onto the planar substrate, the structure can be formed into any curvilinear surface required for the specific application, through a vacuum forming machine. In this process the sample to be formed is first heated to its softening temperature, by using an infrared panel heater. The created mould is, first, pushed into contact with the curved object; and, then, vacuum is applied. The resulting imbalance of air pressure on either side of the substrate results in it taking the shape of the object on which the metasurface is applied. Printed samples were formed, giving two slightly different geometries due to the thickness of the substrate: the conductive grid (metallic) facing towards and the dielectric counterpart facing away, from the mould. A porous breather tissue was positioned around the base of the mould to prevent the substrate from forming a gas seal around the edge of it during forming locking off the vacuum pressure and preventing any further deformation of the substrate into the shape of the mould.

By following the design (formulas (3) and (6) and manufacturing procedure, both Split-Ring-Resonator (SRR, Fig.2(c)) and Complementary SRR (CSRR, Fig.2(d)) have been realized. The unit-cell is formed by square patches with different sizes (side length $l=\lambda_{0} / 15$, strip width $w=\lambda_{0} / 75$ and thickness $t=\lambda_{0} / 30$ ) printed on a grounded dielectric slab (with uniform permittivity value $\varepsilon_{\mathrm{r}}=15$ and thickness $\left.s=\lambda_{0} / 10\right)$. Both dimensions and spatial periodicity are assumed to be smaller than the operative wavelength $\lambda_{0}$. (a)

(c)
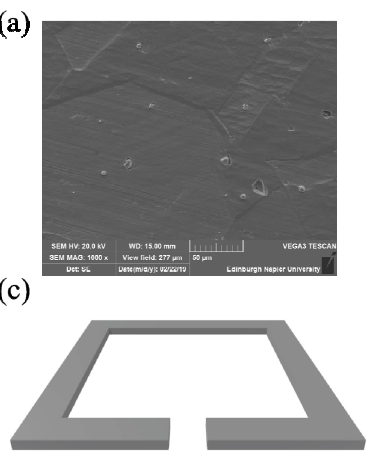

(b)

(d)
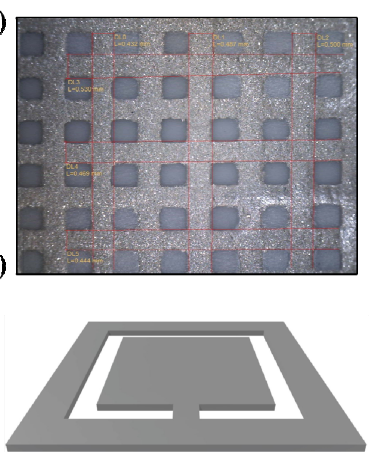

Fig. 2. (a) SEM image of manufactured substrate; (b) SEM image of manufactured metasurface; Square-shape configurations used: (c) SplitRing-Resonator (SRR); (d) Complimentary Split-Ring-Resonator (CSRR).

\section{Experimental Setups and Data Acquisition}

In order to characterize and test the realized metasurface, the following experimental setups have been used [9]:

(i) Absorption measurements: the metasurface is placed at a specific distance from the sample. Changes are detected in the transmission coefficient amplitude and bandwidth, while its frequency position does not change. From the resonance magnitude and amplitude width, we can detect both sample type and concentration.

(ii) Refractive Index measurements. Once the biological sample is placed in contact with the metasurface, the reflection coefficient characteristics (frequency position and amplitude) change as a function of the sample properties. From the frequency position of the resonant dip, it is possible to discern the kind of substance among others.

(iii) Surface measurements. A Near Field scanner is used and programmed to follow the contours of the structure. The probe is positioned very close $(0.5 \mathrm{~mm})$ above the surface, normal to the flat region of the device: only components parallel to the orientation of the probe are 
detected.

The excitation is provided by a E-plane pyramid horn antenna attached to a network analyzer (Agilent N5230C PNA-L), representing the input port 1 . The metasurface is surrounded on its edges by an absorbing layer $\left(\varepsilon_{\mathrm{r}}=3.8-\right.$ j7.2) to reduce reflections at the boundaries. The receiving antenna is attached the network analyzer, representing output port 2. With this 2-port set up, both reflection (S11) and transmission (S21) coefficients are measured.

To scan the surface regions, an NSI planar scanner is programmed to follow the contours of the surface under investigation. Measurements have been done at discrete points along the devices (resolution of $1 \mathrm{~mm} \times 1 \mathrm{~mm}$ ) from which the corresponding amplitude and phase of the electric field component normal to the plane $(\mathrm{Ez})$ are acquired.

\section{RESULTS \& DISCUSSION}

We now use the realized metasurfaces for sensing and medical diagnostics applications. The sensor consists in SRR or CSRR metasurfaces, whose frequency response is modified by the surrounding environment (the biological sample) changes. Biological tissue properties and their frequency response are the results of the interaction between the electromagnetic radiation and their constituents at molecular and cellular level [19]. Diseases typically induce structural, biochemical and mechanical changes, leading to significantly different EM properties in terms of sample permittivity and/or permeability. The metasurfaces reveal such differences, by correlating sample biological changes to its response characteristics. The output signal will strictly depend on such modifications: amplitude, phase, frequency, polarization. (a)

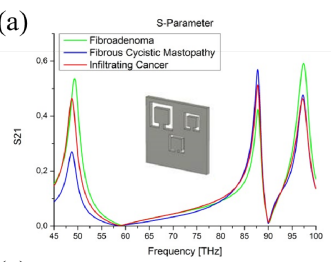

(c)

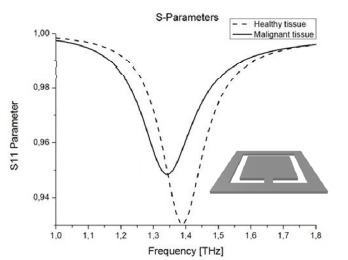

(b)

(d)
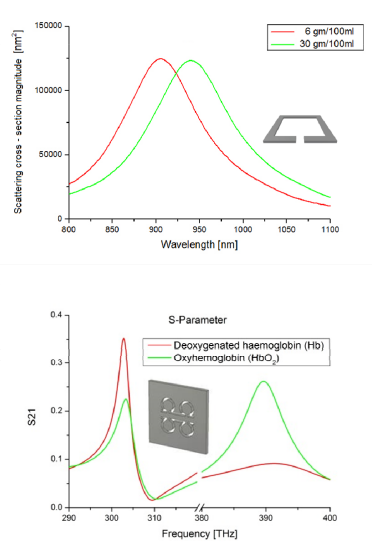

Fig. 3. (a) Breast cancer stage recognition (b) Sugar \& Glucose concentration measurements; (c) Skin cancer detection (d) Blood oxygen level measurements: $\mathrm{HbO}_{2} / \mathrm{Hb}$ ratio.

\section{A. Absorption Measurements}

Resonant cavities/absorbers are of great interest in medical diagnostics to develop highly selective sensors [15]. Organic and inorganic compounds absorb in specific spectral regions. This property gives each material a unique signature in the electromagnetic spectrum, depending on the related molecular structure [20]. Thus, the specificity and uniqueness of such spectra can be used for the recognition of the biological tissue under test. In this case, it is necessary to irradiate the sample in a selective way, to excite only the chemical groups of our interest, leaving unaltered the others.

CSRRs are useful to design high selectivity sensors:

(1) the electromagnetic field has a different configuration pattern compared to the traditional ones. Electric and magnetic field components are not only localized in specific spots like the SRR gaps or its thin strip surface. In CSRR the field lines are present also all along the metal surface plate: the sensing area is greater, ensuring a much stronger wavesample interaction.

(2) They can illuminate the sample in a selective way: it is possible to design the CSRR sensor response for the recognition of specific compounds, by tuning its frequency(ies) with the absorption peak(s) of the sample.

By using eqn. (6.1) and the absorption measurement setup, a metasurface-based sensor has been realized for cancer stage recognition [21]. The sensor consists of a planar CSRR made of circular inclusions. The resonant frequencies of the sensor $(50,87$, and $99 \mathrm{THz})$ are designed to coincide with the proteins and lipids spectral absorption characteristics of three breast sample tissues: infiltrating cancer (red), fibrous-cystic mastopathy (blue), and fibroadenoma (green). The transmission coefficient peaks of the CSRR structure change both magnitude and amplitude width, accordingly to the molecular bonds absorption rate of the considered tissues at such frequencies (Fig.3(a)).

\section{B. Refractive index measurements}

Substrate modes can bring lots of advantages in biological sensing applications, as the field is confined to a very narrow area, that region results to be very sensitive to any changes, even the smallest ones. Any molecules can alter the total effective refractive index, and therefore change the sensor response [16]. Refractive index of solutions increases with the increasing of the chemical species concentration. Therefore, it would be possible to sense the presence of either organic or inorganic compounds [22]. For an accurate detection of such substances, a huge electric field enhancement can be obtained by designing the related sensing area (eqn. 6.2). Practical applications have been proposed for sugar and glucose measurements [23]. A highly sensitive and selective sugar detection sensor was designed and realized by using SRR metasurfaces. The strongly localized and enhanced transmission obtained in the substrate area highly increase the molecular absorption to allow the selective detection of D-glucose and sucrose at low concentrations (Fig.3(b)). Thanks to its high sensitivity (30k $\mathrm{nm} / \mathrm{RIU})$, this device holds the potential for further practical applications to be fabricated as a lab-on-a-chip device.

\section{Surface-waves Measurements}

Surface-wave measurements are crucial for sensing applications where it is important to carry stronger signals with higher bandwidth and transmission rates as compared to their counterparts such as optical fibers [17]. Thanks to the 
signal amplification effects caused by the surface waves in metasurfaces, both SRR and CSRR structures have been realized for:

(i) Skin cancer recognition. Structural modifications (size, shape) of chromophores and pigments produce variations of the skin absorption properties [24]. In [25] the sensor consists of multilayered resonating inclusions arranged in a planar array configuration. The possibility to tune the sensing structure resonances with such spectral characteristics allows us to identify specific diseases (Fig.3(c)).

(ii) Oxygen levels in blood. Hemoglobin is responsible for transporting oxygen, carried by human blood, to several tissues and organs, where the oxygen can be used by other cells [26]. The absorption spectra of oxyhemoglobin $\left(\mathrm{HbO}_{2}\right)$ and deoxyhemoglobin $(\mathrm{Hb})$ are much different. This difference is used to measure the amount of oxygen in patient's blood. In [27] the structure consists of a multiple square CSRR particles in a single unit-cell, having its resonant frequencies coincident with the $\mathrm{HbO} 2$ and $\mathrm{Hb}$ absorption frequencies. The transmission coefficient (magnitude and bandwidth) changes accordingly to the sample absorption rates. $\mathrm{HbO} 2$ has its lower absorption at $660 \mathrm{~nm}(400 \mathrm{THz})$ compared to $\mathrm{Hb}$ having its higher absorption at $940 \mathrm{~nm}(315 \mathrm{THz})$, as shown in Fig.3(d).

\section{CONCLUSIONS}

Metasurface-based sensors have been manufactured and practically tested for medical diagnostic applications.

The design method, here proposed, permits to obtain full control of the sensor response; most importantly by using this approach, the user can manipulate at will the sensor features accordingly to the required application.

Metasurfaces fabrication processes are usually expensive and restricted to a small area, and not easily scalable, especially for complex shapes. The reason is due to the fine size required to operate at the wavelength used in the specific application. In this work, instead, we fabricated the sample by producing the mesh first, then formed the shape post mesh deposition, as dictated by the proposed approach. This brings two main advantages: $(i)$ full control of both size and shape during the fabrication stage; (ii) high reproducibility (different frequency ranges) and scalability (different shape and geometry used).

Such characteristics have been confirmed, also, by the experimental tests carried out on both the SRR and CSRR configurations: sugar level recognition, cancer detection and oxygen levels measurements.

Metasurfaces showed high performances in terms of sensitivity and selectivity, crucial properties to use them as sensors in different areas of medical diagnostics. This pave the way for their implementation far beyond healthcare sector, in other industrial applications, such as: food technology, safety, forensics and military.

\section{REFERENCES}

[1] J. Fraden, Handbook of Modern Sensors: Physics, Designs, and Applications, Springer, San Diego, CA, USA, 2015

[2] J. G. Webster, H. Eren, Measurement, Instrumentation, and Sensors Handbook, CRC Press, Taylor \& Francis Group, 2018

[3] X. C. Tong, Functional Metamaterials and Metadevices, Springer, Bolingbrook, IL, USA, 2017

[4] N.I. Zheludev and Y.S. Kivshar, "From metamaterials to metadevices," Nature Materials 11,917-924, 2012.

[5] H.-T. Chen, A.J. Taylor and N. Yu, "A review of metasurfaces: physics and applications," Reports on Progress in Physics, 79, 076401, 2016.

[6] F. Monticone, N. M. Estakhri, A. Alù, "Full control of nanoscale optical transmission with a composite metascreen," Phys. Rev. Lett. 110, 203903, 2013.

[7] Qin, F, et al., "Hybrid bilayer plasmonic metasurface efficiently manipulates visible light," Sci. Adv., 2, 1501168, 2016.

[8] L. P. Eisenhart, Separable systems in euclidean 3-space. Phys. Rev. $1934,45,427$.

[9] L. LaSpada, "Metasurfaces for Advanced Sensing and Diagnostics," Sensors 19(2), 355, 2019.

[10] P. Moon and D. E. Spencer, Field Theory Handbook, Berlin, Germany, Springer-Verlag (1963)

[11] C.A. Balanis, Advanced Engineering Electromagnetic, 4th Edition, Wiley, 2004.

[12] P. Gay-Balmaz and O.J.F. Martin, "Electromagnetic resonances in individual and coupled split-ring resonators," Journal of Applied Physics 92, 2929, 2002.

[13] J. B. Pendry, et al. "Magnetism from conductors and enhanced nonlinear phenomena," IEEE Transactions on Microwave Theory and Techniques, 47(11), 2075-2084, 1999.

[14] M A Bueno and A K T Assis, "A new method for inductance calculations,” J. Phys. D: Appl. Phys. 28, 1802, 1995

[15] L La Spada, L Vegni, Metamaterial-based wideband electromagnetic wave absorber, Optics express 24 (6), 5763-5772, 2016.

[16] L La Spada, L Vegni, Near-zero-index wires, Optics express 25 (20), 23699-23708, 2017.

[17] L. La Spada, et al., "Curvilinear MetaSurfaces for Surface Wave Manipulation, Sci. Rep. 9, 3107, 2019

[18] L. La Spada, et al. "Surface Wave Cloak from Graded Refractive Index Nanocomposites," Sci. Rep., 6, 29363, 2016.

[19] Gabriel, S. The dielectric properties of biological tissues. Phys. Med. Biol. 41, 2271-2293, 1996.

[20] ME Candela, et al., "Resident mesenchymal progenitors of articular cartilage. Matrix Biology," 39, 44-49, 2014

[21] R Iovine, et al., Optical properties of modified nanorod particles for biomedical sensing, IEEE Transactions on Magnetics 50 (2), 169-172, 2014.

[22] S. Asai, et al., "Tendon Progenitor Cells in Injured Tendons Have Strong Chondrogenic Potential: The CD 105 Negative Subpopulation Induces Chondrogenic Degeneration. Stem Cells," 32 (12), 3266-3277, 2014.

[23] L La Spada, L Vegni, Electromagnetic nanoparticles for sensing and medical diagnostic applications, Materials 11 (4), 603, 2018.

[24] Salomatina, E.V.; Jiang, B.; Novak, J.; Yaroslavsky, A.N. Optical properties of normal and cancerous human skin in the visible and near-infrared spectral range. J. Biomed. Opt., 11, 064026, 2006.

[25] L La Spada, et al., Metamaterial biosensor for cancer detection, IEEE Sensors, 627-630, 2011

[26] Wiernik, P.H.; Dutcher, J.P.; Gertz, M.A. Neoplastic Diseases of the Blood; Springer: Berlin, Germany, 2018.

[27] TM McManus, et al., Isotropic and anisotropic surface wave cloaking techniques, Journal of Optics 18 (4), 044005, 2016. 